\title{
An instrument for low- and variable-temperature millimeter-wave surface impedance measurements under magnetic fields
}

\author{
Tetsuo Hanaguria) \\ Department of Advanced Materials Science, The University of Tokyo, 7-3-1, Hongo, Bunkyo-ku, \\ Tokyo 113-0033 and SORST, Japan Science and Technology Corporation, 4-1-8, Honcho, Kawaguchi, \\ Saitama 332-0012, Japan \\ Keishi Takaki ${ }^{\text {b) }}$ \\ Department of Advanced Materials Science, The University of Tokyo, 7-3-1, Hongo, Bunkyo-ku, \\ Tokyo 113-0033, Japan \\ Yoshishige Tsuchiya ${ }^{\mathrm{c})}$ and Atsutaka Maeda \\ Department of Basic Science, The University of Tokyo, 3-8-1, Komaba, Meguro-ku, Tokyo 153-8902, Japan
}

(Received 28 April 2003; accepted 25 June 2003)

\begin{abstract}
We describe a low-temperature millimeter-wave (44 GHz) surface impedance measurement instrument based on the cavity perturbation method. In this instrument, all millimeter-wave paths at low temperatures (including the cavity resonator), are located inside a high vacuum tube, which can be inserted into a $7 \mathrm{~T}$ superconducting magnet. This design, which is free of exchange gas, enables measurements over a wide temperature range as well as providing stable and reproducible operation. By pumping a ${ }^{3} \mathrm{He}$ pot attached to a cavity, a sample can be cooled well below $1 \mathrm{~K}$. We present experimental results which demonstrate the performance of the instrument. (C) 2003 American Institute of Physics. [DOI: 10.1063/1.1606539]
\end{abstract}

\section{INTRODUCTION}

Measurements of the high frequency electrodynamic response of solids play an important role in condensed matter physics. For example, the magnetoresonance absorption of electromagnetic waves at microwave and millimeter-wave frequencies leads to the techniques of electron spin resonance and cyclotron resonance. Besides these resonance measurements, the complex conductivity at high frequencies and low temperatures also provides rich information. Exotic materials such as charge/spin density wave systems, ${ }^{1,2}$ heavy fermion compounds, ${ }^{3}$ and transition metal oxides ${ }^{4}$ often have low energy collective excitations which characterize their ground states. Microwave and millimeter-wave complex conductivity measurements are indispensable to investigate the details of such excitations since their characteristic energies often lie in regions where standard optical spectroscopy is impossible. Complex conductivity measurements also play an important role in superconductivity research. Information on quasiparticles, superfluids, and vortex dynamics can be extracted from the complex conductivity. ${ }^{5-7}$

The most important quantity in magnetoresonance measurements is the strength of the magnetic field at which the resonance occurs, while the absolute value of the absorption is of secondary importance. However, in the case of complex conductivity measurements, the absolute value of the com-

\footnotetext{
a) Author to whom correspondence should be addressed; electronic mail: hanaguri@k.u-tokyo.ac.jp

b) Present address: Yokohama R\&D Laboratories, The Furukawa Electric Co., Ltd., 2-4-3, Okano, Nishi-ku, Yokohama 220-0073, Japan.

${ }^{c}$ Present address: Research Center for Quantum Effect Electronics, Tokyo Institute of Technology, 2-12-1, O-okayama, Meguro-ku, Tokyo 152-8552, Japan.
}

plex response function, such as the surface impedance, is of primary importance. In both cases, measurements are generally made using the cavity perturbation method. ${ }^{8}$ The complex response function is obtained by measuring both the quality factor $Q$ and the resonance frequency $f$ of the cavity, correcting for the values of the blank cavity. Therefore, for complex conductivity measurements, the instrument should be highly stable despite repetitive opening and closing of the cavity for exchange of the sample and possible changes in the environment during the measurements. These restrictions are somewhat relaxed for magnetoresonance measurements. Here, we describe the design and performance of a highly stable millimeter-wave surface impedance measurement instrument based on the cavity perturbation method. Our instrument can operate over a very wide range of temperatures from 0.5 to above $100 \mathrm{~K}$, and in magnetic fields up to $7 \mathrm{~T}$.

\section{INSTRUMENTATION}

\section{A. Low-temperature insert}

At low temperatures, there are several factors which reduce the stability of a millimeter-wave measurement system. First, any exchange gas used to control the thermal link between the sample and the cryogen reduces the reproducibility of the background $Q$ and $f$. This is because it is difficult to reproducibly control the pressure of the exchange gas, and hence its dielectric constant. Second, direct immersion of the waveguide in the cryogen makes the measurements sensitive to the cryogen level. To remove these problems, we put all the millimeter-wave paths inside the vacuum tube, which is 


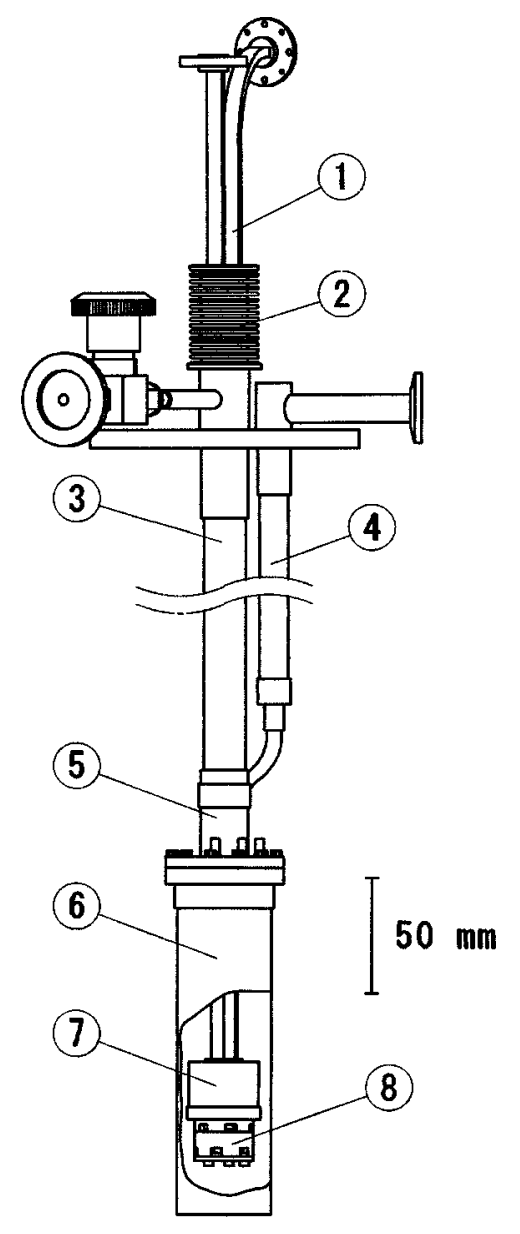

FIG. 1. Schematic of the low temperature insert: (1) waveguides, (2) bellows, (3) evacuation tube, (4) ${ }^{3} \mathrm{He}$ pumping line, (5) radiation trap, (6) vacuum can, (7) ${ }^{3} \mathrm{He}$ pot, and (8) cavity resonator.

evacuated to a vacuum better than $10^{-6}$ Torr. Such a design, free of exchange gas, also enables us to operate the instrument over a very wide temperature range.

Figure 1 shows a schematic illustration of the lowtemperature insert, which can be placed in a liquid He Dewar with a 7 T superconducting magnet. ${ }^{9}$ A cavity resonator (8) is located inside the vacuum can (6) and tightly bolted onto a ${ }^{3} \mathrm{He}$ pot (7). Two $\mathrm{Cu}-\mathrm{Ni}$ waveguides (1) are connected to the cavity in order to introduce and transmit the millimeter-wave signals. The waveguides run inside the thin-wall stainlesssteel tube (3) used to evacuate the vacuum can, and do not directly contact with liquid He. To absorb the difference in thermal contraction between the stainless-steel tube and the waveguides, we put bellows (2) on the top flange of the insert. In this design, the waveguides are mechanically and thermally fixed at two well defined positions: the top of the bellows and the radiation trap (5) at the bottom of the stainless-steel tube. During a measurement, the temperatures at these two positions are kept constant at room temperature and liquid He temperature, respectively. Therefore, the environment around the waveguides does not change at all, even if the liquid He level changes.

To achieve temperatures below $1 \mathrm{~K}$, the radiation from room temperature must be carefully trapped. The structure of the radiation trap is illustrated in Fig. 2. The waveguides (3)

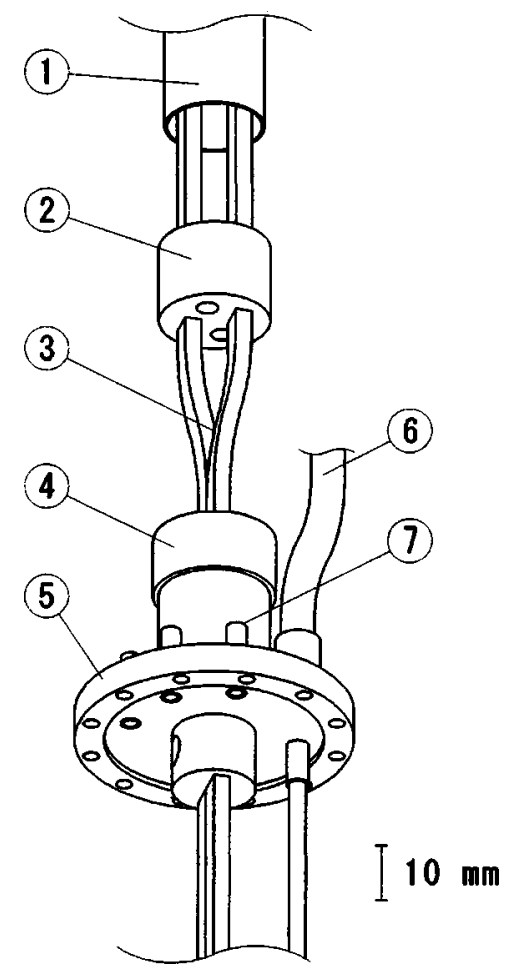

FIG. 2. Schematic of the radiation trap: (1) evacuation tube, (2) upper OFC plug, (3) waveguides, (4) lower OFC plug, (5) flange of the vacuum can, (6) ${ }^{3} \mathrm{He}$ pumping line, and (7) feedthrough for the wires.

are bent into $\mathrm{S}$ shapes and the top and bottom of each bend are silver soldered to plugs made of oxygen-free copper (OFC) [(2) and (4)]. These plugs are in direct contact with liquid $\mathrm{He}$ to cool the bend and therefore trap the radiation.

\section{B. Cavity resonator and detection scheme}

The design of the cavity resonator depends on the exact nature of the measurement. Here, we focus on a resonator suitable for measurements on metallic samples in the skin depth regime. ${ }^{8}$ In this case, the relevant property is the surface impedance $Z_{s}=R_{s}+i X_{s}$. The surface resistance $R_{s}$ and the surface reactance $X_{s}$ are related to $Q$ and $f$ by

$$
\begin{aligned}
R_{S} & =G\left(\frac{1}{2 Q}-\frac{1}{2 Q_{B}}\right), \\
X_{s} & =-G\left(\frac{f-f_{B}}{f_{B}}\right)+C, \\
& \sim-G\left(\frac{\Delta f}{f^{0}}-\frac{\Delta f_{B}}{f_{B}^{0}}\right)+C^{\prime} .
\end{aligned}
$$

Here, $Q_{B}$ and $f_{B}$ are the blank cavity values of $Q$ and $f$, respectively. $\Delta f$ and $\Delta f_{B}$ are the resonance frequency shifts caused by the change in temperature/magnetic field. $f^{0}$ and $f_{B}^{0}$ are the reference frequencies from which $\Delta f$ and $\Delta f_{B}$ are measured. $G, C$, and $C^{\prime}$ are constants determined by the geometries of the cavity and the sample. $C$ and $C^{\prime}$ differ by $G\left(f^{0}-f_{B}^{0}\right) / f_{B}^{0}$. One can calculate the complex conductivity $\sigma=\sigma_{1}-i \sigma_{2}$ using 


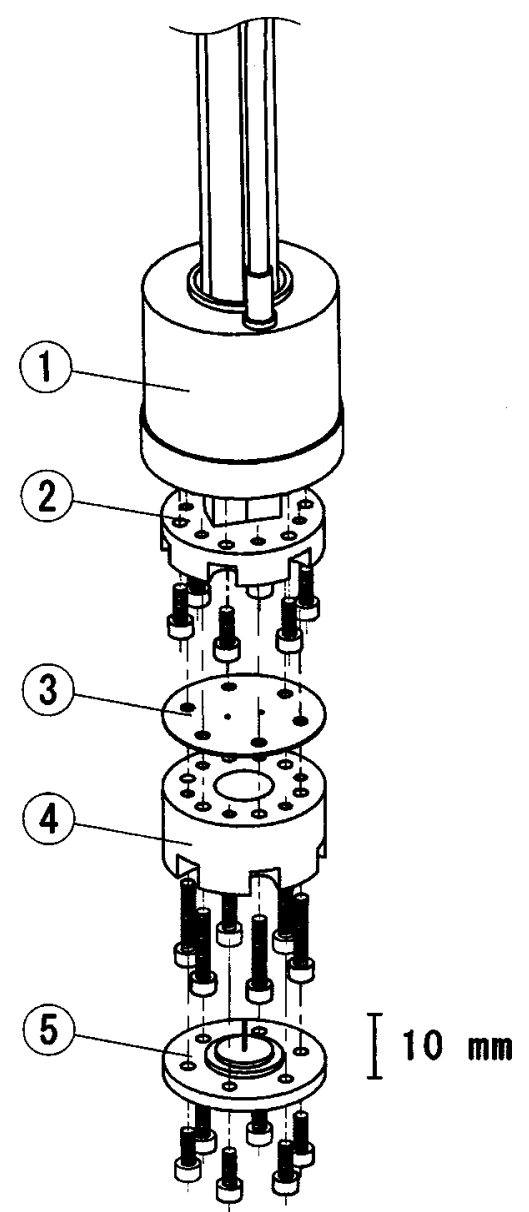

FIG. 3. Schematic of the ${ }^{3} \mathrm{He}$ pot and the cavity resonator: (1) ${ }^{3} \mathrm{He}$ pot, (2) end flange for waveguides, (3) cavity top plate, (4) cavity body, and (5) end plate with a sapphire pin.

$$
\begin{aligned}
& \sigma_{1}=\mu_{0} \omega \frac{2 R_{s} X_{s}}{\left(R_{s}^{2}+X_{s}^{2}\right)^{2}}, \\
& \sigma_{2}=\mu_{0} \omega \frac{X_{s}^{2}-R_{s}^{2}}{\left(R_{s}^{2}+X_{s}^{2}\right)^{2}},
\end{aligned}
$$

and the complex resistivity $\rho=\rho_{1}+i \rho_{2}$ using

$$
\begin{gathered}
\rho_{1}=\frac{2 R_{s} X_{s}}{\mu_{0} \omega}, \\
\rho_{2}=\frac{X_{s}^{2}-R_{s}^{2}}{\mu_{0} \omega} .
\end{gathered}
$$

Here, $\mu_{0}$ is the vacuum permeability and $\omega=2 \pi f$.

For typical measurements, the sample is located at the antinode of the millimeter-wave magnetic field inside the cavity. We adopted a cylindrical cavity which resonates in the $\mathrm{TE}_{011}$ mode because of its high $Q_{B}$ and the ease of machining. For surface impedance measurements at low temperatures, the cavity should fulfill the following requirements. First, the cavity should be thermally connected to the ${ }^{3} \mathrm{He}$ pot for effective cooling. Second, it should be removable from the insert in order to re-polish or replace the cavity. Finally, the cavity should be stable after repetitive opening and closing to exchange the sample. Based on these factors, we designed the cavity as illustrated in Fig. 3. It consists of

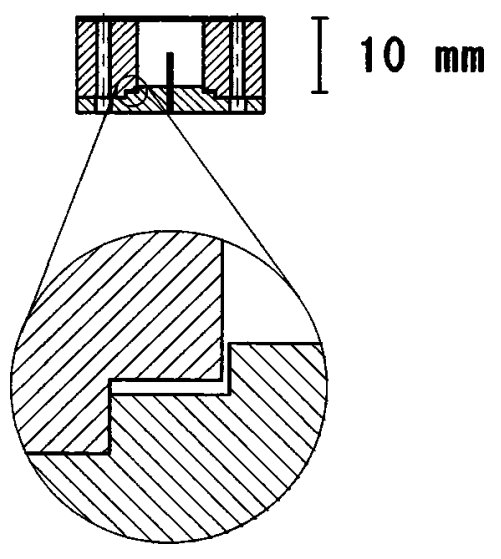

FIG. 4. Cross section of the cavity resonator. A magnified view of the joint between the cavity body and the end plate is also shown.

three parts: a $0.25 \mathrm{~mm}$ thick top plate (3), the cavity body (4), and an end plate (5). Both the inner diameter and the height of the cavity are $9 \mathrm{~mm}$, giving a resonant frequency of $44 \mathrm{GHz}$. All the parts are made of OFC and the inner wall was carefully polished with a polishing paste. ${ }^{10}$ Coupling to the waveguides occurs through two slits in the top plate, their locations crossing the antinodal line of the millimeter-wave currents. To reconcile the high coupling $Q$ (which requires small slits) and the sufficient signal intensity (which demands large slits), $Q_{B}$ is tuned to be $1.2 \times 10^{4}$ at room temperature and reaches $2.6 \times 10^{4}$ at $4.2 \mathrm{~K}$.

The incident and transmission waveguides end at the OFC flange (2) on which the cavity is bolted. To minimize the direct coupling between the two waveguides, we sandwiched a thin indium sheet between the end flange and the top plate of the cavity. The end flange is tightly bolted on the bottom of the OFC ${ }^{3} \mathrm{He}$ pot to ensure good thermal contact. All the screws can be accessed from the bottom and thus the cavity can be easily removed from the insert.

The sample is placed at the center of the cavity (the antinode of the millimeter-wave magnetic field) by mounting on a $0.5 \mathrm{~mm}$ diam sapphire rod using a small amount of Apiezon $\mathrm{N}$ grease. ${ }^{11}$ The other end of the sapphire rod is inserted into the hole which passes through the center of the end plate and is epoxide using Stycast $1266 .{ }^{12}$ The radiation loss from the hole is negligible because there is essentially no current crossing the hole.

To change the sample, the end plate needs to be removed from the body of the cavity. To remove any irreproducibility associated with this process, we adopt the following design. As shown in Fig. 4, the end plate has stepped mounds. The bottom of the cavity body is spot faced to accept the outer mound of the end plate with little play between them. Therefore, the sapphire rod can be located exactly at the center of the cavity in a reproducible manner. The inner mound works as a mode trap which removes the degeneracy between $\mathrm{TE}_{011}$ and $\mathrm{TM}_{111}$ modes. The end plate is bolted on the cavity body by six M2 screws with a torque of $1.5 \mathrm{kgf} \mathrm{cm}$.

The values of $Q$ and $f$ for the cavity are obtained by fitting the resonance curve to a Lorentzian at each temperature/magnetic field. The resonance curve is measured in the standard transmission configuration. Input signals are 
supplied from a synthesized sweeper with a millimeter-wave source module ${ }^{13}$ and are fed into the incident waveguide. A portion of the supplied signal is separated by a directional coupler and used as a reference. The transmitted and reference signals are detected and the power ratio between them is measured as a function of frequency by a scalar network analyzer. ${ }^{14}$ We typically use a frequency span of about 1.5 times the full width at half maximum of the resonance curve. All the systems are controlled via general purpose interface bus.

\section{Cooling scheme and temperature regulation}

The sample and cavity are cooled together through the thermal connection provided by the sapphire rod. By pumping the main He bath with a $1200 \mathrm{l} / \mathrm{min}$ rotary pump, we can reach temperatures down to $1.5 \mathrm{~K} .{ }^{15}$ To go below $1.5 \mathrm{~K}, 51$ of ${ }^{3} \mathrm{He}$ gas are liquefied within the ${ }^{3} \mathrm{He}$ pot, which is then pumped by a $200 \mathrm{l} / \mathrm{min}$ rotary pump through a $12 \mathrm{~mm}$ diameter thin-wall stainless-steel tube. The lowest attainable temperature is about $0.5 \mathrm{~K}$ and the ${ }^{3} \mathrm{He}$ duration time is about 3 $\mathrm{h}$. In order to check the temperature difference between the sample and the cavity, we measured the resonant frequency of the cavity when loaded with zinc and thus evaluated its superconducting transition temperature $T_{c}$. At an incident millimeter-wave power of $-12 \mathrm{~dB} \mathrm{~m}$, the measured $T_{c}$ was $0.72 \mathrm{~K}$, which is lower than the standard value of $0.85 \mathrm{~K}$. This difference can be attributed to the remanent field of the superconducting magnet and the self heating of the sample. By examining the millimeter-wave power dependence, we estimated that the effect of the self-heating is about $15 \mathrm{mK}$ at $-12 \mathrm{~dB} \mathrm{~m}$. This value can be reduced by using a lower incident power, in which case, a low-noise amplifier in the transmission path is required to enhance the signal-to-noise ratio.

The temperature of the cavity is monitored and regulated by a calibrated Cernox thermometer ${ }^{16}$ and a manganin wire heater wound around the ${ }^{3} \mathrm{He}$ pot. Using a commercial temperature controller, ${ }^{17}$ temperature stability of the order of \pm 1 $\mathrm{mK}$ may be achieved at temperatures from 0.5 to above 100 $\mathrm{K}$. The heater power needed to keep the sample at $100 \mathrm{~K}$ is about $0.1 \mathrm{~W}$. Measurements at higher temperatures, even at room temperature, are possible, although naturally the liquid He consumption is increased.

\section{RESULTS}

\section{A. Stability of the cavity}

Figure 5 shows the temperature dependence of the blank cavity parameters, $1 / 2 Q_{B}$ and $\Delta f_{B} / f_{B}^{0}$. The reference frequencies $f_{B}^{0}$, s are the resonance frequencies at the lowest temperature. Because of the thermal expansion of the cavity, $\Delta f_{B} / f_{B}^{0}$ shows rather large temperature dependence. The plot shows the results of 5 different experimental runs over a period of more than $1 \mathrm{yr}$. Between the runs, the system was warmed up and the cavity opened. Despite such treatment, the reproducibility of the data is quite good. ${ }^{18}$ To estimate the run-to-run variations in more detail, the background temperature dependence should be subtracted. To do this, we

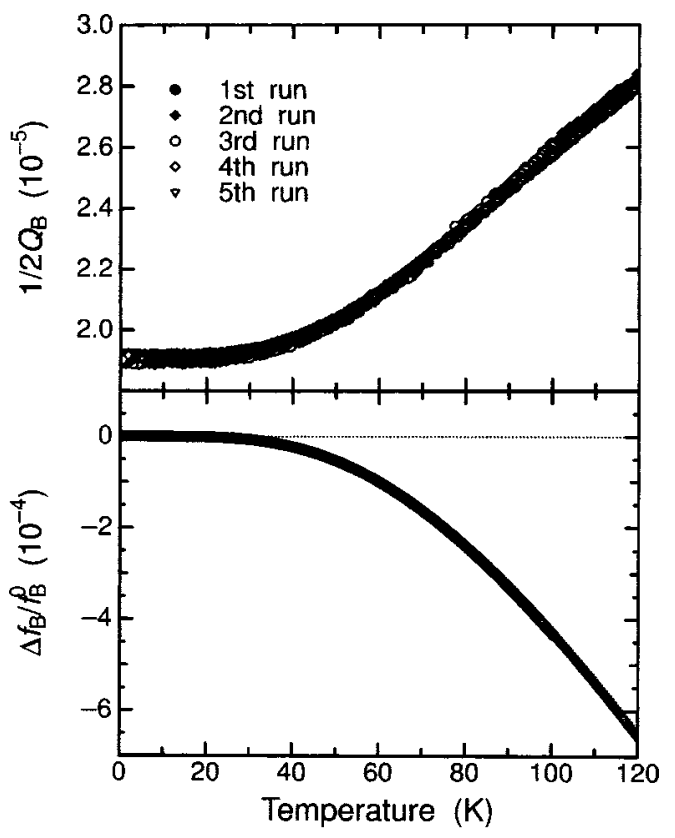

FIG. 5. Temperature dependence of the blank cavity parameters $1 / 2 Q_{B}$ and $\Delta f_{B} / f_{B}^{0}$. Results of five independent runs are plotted.

used a polynomial fit to all the data for $1 / 2 Q_{B}$ or $\Delta f_{B} / f_{B}^{0}$ and subtracted it from the data from each experimental run. The deviations from the polynomial are labeled as $\delta\left(1 / 2 Q_{B}\right)$ or $\delta\left(\Delta f_{B} / f_{B}^{0}\right)$. We found that $\left|\delta\left(1 / 2 Q_{B}\right)\right|<3.0 \times 10^{-7}$ and $\left|\delta\left(\Delta f_{B} / f_{B}^{0}\right)\right|<1.0 \times 10^{-6}$ up to $120 \mathrm{~K}$. Below $40 \mathrm{~K}$, the reproducibility of $\Delta f_{B} / f_{B}^{0}$ increases because of the decrease in the thermal expansion coefficient. Overall, both $\delta\left(1 / 2 Q_{B}\right)$ and $\delta\left(\Delta f_{B} / f_{B}^{0}\right)$ are comparable to the scatter of the data at each run $\left(\sim 1.0 \times 10^{-7}\right)$.

We also checked the magnetic-field dependence of $1 / 2 Q_{B}$ and $\Delta f_{B} / f_{B}^{0}$. There was no sign of any parasitic signals, such as electron spin resonances from the paramagnetic impurities in the sapphire rod. The parameters for the blank cavity show weak monotonic magnetic-field dependence $\left(1 / 2 Q_{B}(0.5 \mathrm{~K}, 7 \mathrm{~T})-1 / 2 Q_{B}(0.5 \mathrm{~K}, 0 \mathrm{~T}) \sim-4.0\right.$ $\times 10^{-7}, \Delta f_{B}(0.5 \mathrm{~K}, 7 \mathrm{~T}) / f_{B}^{0}-\Delta f_{B}(0.5 \mathrm{~K}, 0 \mathrm{~T}) / f_{B}^{0} \sim 3.0$ $\times 10^{-7}$ ) and the changes become smaller with increasing temperature. Although the origin of the magnetic-field dependence is unclear, the blank cavity parameters are sufficiently stable, with run-to-run variations of less than 1.0 $\times 10^{-7}$ in magnetic fields up to $7 \mathrm{~T}$.

\section{B. Application to a superconductor}

The instrument was tested on the superconductor $\mathrm{Nb}_{0.8} \mathrm{Ta}_{0.2} \mathrm{Se}_{2}$. This material is a dirty $s$-wave superconductor, in which conventional behavior is expected. The sample was cut into a rectangular shape with dimensions of $0.8 \times 0.8 \times 0.1 \mathrm{~mm}^{3}$ with the shortest dimension along the $c$ axis. Millimeter-wave magnetic fields were applied along the $c$ axis.

Figure 6 shows the temperature dependence of $1 / 2 Q$ and $\Delta f / f^{0}$ for the cavity containing the sample. We also plot the blank data presented in Fig. 5 as a reference. The values of $\Delta f$ and $\Delta f_{B}$ are measured relative to the resonance frequencies at the lowest temperature. It is clear that the run-to-run 


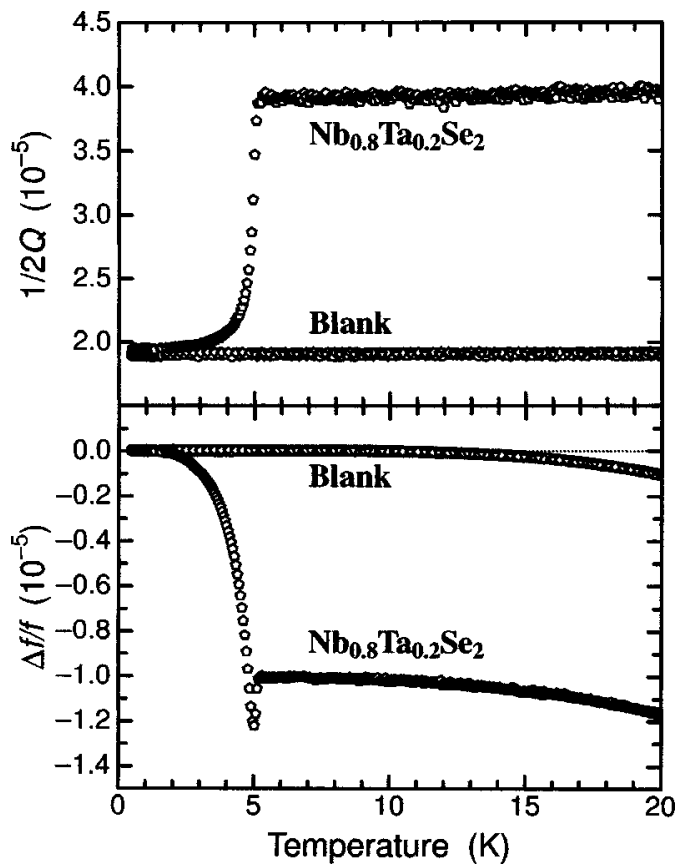

FIG. 6. Temperature dependence of $1 / 2 Q$ and $\Delta f / f^{0}$ of the cavity loaded with $\mathrm{Nb}_{0.8} \mathrm{Ta}_{0.2} \mathrm{Se}_{2}$. Data of the blank cavity are also plotted for the reference.

variations of the blank cavity parameters are very small compared with the sample signal. Sharp changes in $1 / 2 Q$ and $\Delta f / f^{0}$ indicate a clear superconducting transition at $5.2 \mathrm{~K}$. A spike structure in $\Delta f / f^{0}$ just below $T_{c}$ originates from the competition between the increase in the skin depth and the decrease in the penetration depth with decreasing temperature.

To calculate the absolute value of the surface impedance,

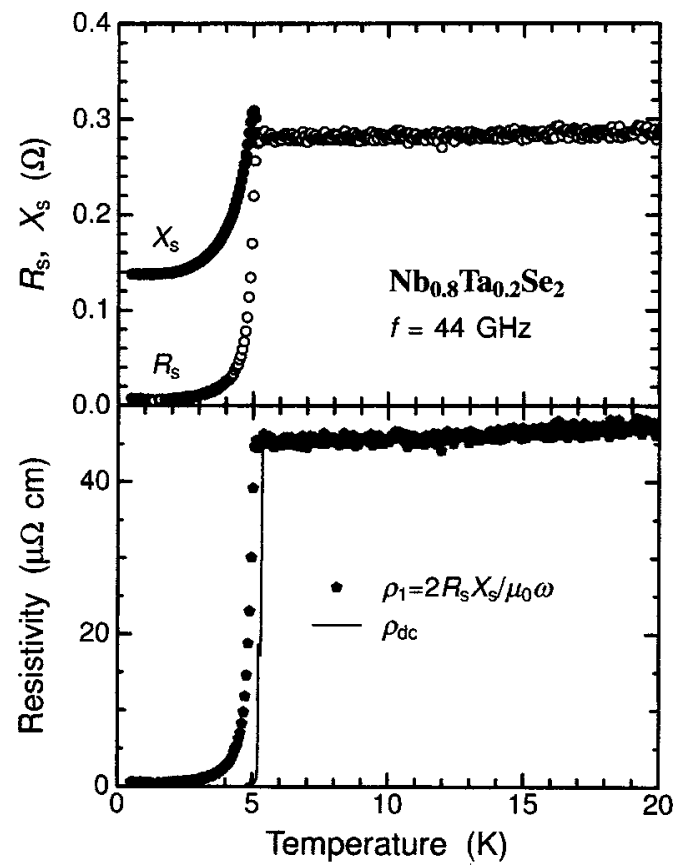

FIG. 7. (Upper panel) Temperature dependence of $R_{s}$ and $X_{s}$ of $\mathrm{Nb}_{0.8} \mathrm{Ta}_{0.2} \mathrm{Se}_{2}$. (Lower panel) Temperature dependence of the real part of the complex resistivity of $\mathrm{Nb}_{0.8} \mathrm{Ta}_{0.2} \mathrm{Se}_{2}$. The dc resistivity is also plotted for reference.

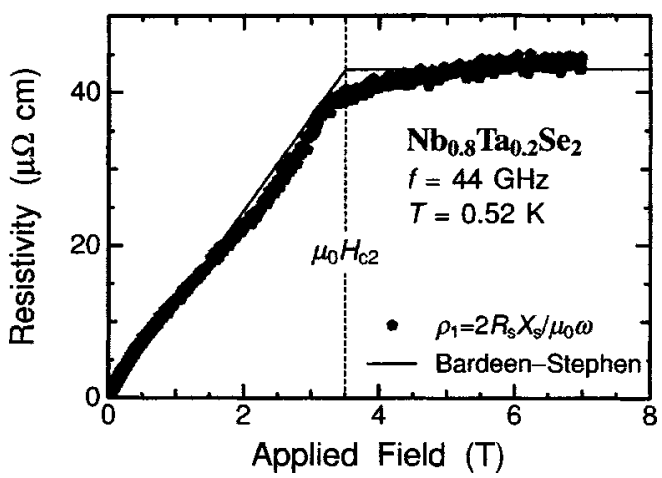

FIG. 8. Magnetic-field dependence of the real part of the complex resistivity of $\mathrm{Nb}_{0.8} \mathrm{Ta}_{0.2} \mathrm{Se}_{2}$ at $0.52 \mathrm{~K}$.

we should evaluate $G$ and $C$ (or $C^{\prime}$ ) in Eqs. (1) and (2). These constants can be estimated from the dc resistivity $\rho_{\mathrm{dc}}$ by assuming the Hagen-Rubens relation, $R_{s}=X_{s}$ $=\sqrt{\mu_{0} \omega \rho_{\mathrm{dc}} / 2}$, above $T_{c}$. The calculated values for $R_{s}, X_{s}$ and $\rho_{1}$ of $\mathrm{Nb}_{0.8} \mathrm{Ta}_{0.2} \mathrm{Se}_{2}$ are plotted in Fig. 7. A very small residual $R_{s}$ is consistent with the bulk superconductivity of the sample. From $X_{s}$ at the lowest temperature, the in-plane London penetration depth $\lambda$ is estimated to be $4000 \AA$. Although a value for $\lambda$ in $\mathrm{Nb}_{0.8} \mathrm{Ta}_{0.2} \mathrm{Se}_{2}$ has not yet been reported, the obtained value seems reasonable considering that the value of $\lambda$ in pure $\mathrm{NbSe}_{2}$ is estimated to be $1600 \AA$ (Ref. 19) and $\lambda$ becomes longer with increasing impurity content. In the superconducting state, our instrument has a resistivity resolution of $0.1 \mu \Omega \mathrm{cm}$.

Next we show the data taken in the presence of magnetic fields. Figure 8 shows the magnetic-field dependence of $\rho_{1}$ at $0.52 \mathrm{~K}$. Both static and millimeter-wave magnetic fields were applied along the $c$ axis. At millimeter-wave frequencies, the effects of vortex pinning can be completely neglected and $\rho_{1}$ then reflects the pure flux flow resistivity. ${ }^{7}$ As expected from the Bardeen-Stephen theory of flux flow in dirty superconductors, ${ }^{20} \rho_{1}$ changes almost linearly with magnetic field and reaches the normal state value at the upper critical field $H_{c 2}$. The estimated $H_{c 2}$ is $3.5 \mathrm{~T}$, which is similar to the value of $3.2 \mathrm{~T}$ estimated from specific heat measurements. ${ }^{21}$

\section{DISCUSSION}

We have described a millimeter-wave surface impedance measurement instrument that works over a very wide temperature range from 0.5 to above $100 \mathrm{~K}$ and in magnetic fields up to $7 \mathrm{~T}$. Very high reproducibility of the blank cavity properties is achieved, for example, $\left|\delta\left(1 / 2 Q_{B}\right)\right|<3.0$ $\times 10^{-7}$ and $\left|\delta\left(\Delta f_{B} / f_{B}^{0}\right)\right|<1.0 \times 10^{-6}$. Below $40 \mathrm{~K}$, the runto-run variations of the blank cavity properties are comparable to the resolution of the measurements. The results presented here demonstrate that our design is very effective for stable and reproducible operation of the cavity resonator. Our design concept can be applied not only to surface impedance measurements but also to other high-frequency measurements based on a cavity perturbation technique. 


\section{ACKNOWLEDGMENTS}

The authors thank S. Komiyama, H. Kitano, and K. Kinoshita for valuable comments and technical assistance. They also thank M. Nohara for providing the $\mathrm{Nb}_{0.8} \mathrm{Ta}_{0.2} \mathrm{Se}_{2}$ crystals and D. G. Steel for a critical reading of the manuscript. This work was partly supported by a Grant-in-Aid for Scientific Research from the Ministry of Education, Science, Sports and Culture of Japan.

${ }^{1}$ G. Grüner, Rev. Mod. Phys. 60, 1129 (1988).

${ }^{2}$ G. Grüner, Rev. Mod. Phys. 66, 1 (1994).

${ }^{3}$ J. R. Feller, C.-C. Tsai, J. B. Ketterson, J. L. Smith, and B. K. Sarma, Phys. Rev. Lett. 88, 247005 (2002).

${ }^{4}$ H. Kitano, R. Inoue, T. Hanaguri, A. Maeda, N. Motoyama, M. Takaba, H. Eisaki, and S. Uchida, Europhys. Lett. 56, 434 (2001).

${ }^{5}$ A. Hosseini, R. Harris, S. Kamal, P. Dosanjh, J. Preston, R. Liang, W. N. Hardy, and D. A. Bonn, Phys. Rev. B 60, 1349 (1999).

${ }^{6}$ N. E. Hussey, Adv. Phys. 51, 1685 (2002) and references therein.

${ }^{7}$ K. Takaki et al., Phys. Rev. B 66, 184511 (2002).
${ }^{8}$ O. Klein, S. Donovan, M. Dressel, and G. Grüner, Int. J. Infrared Millim. Waves 14, 2423 (1993).

${ }^{9}$ Cryogenic Ltd., London, U.K.

${ }^{10}$ Pikal, Nihon Maryo-kogyo Co., Ltd., Tokyo, Japan.

${ }^{11}$ Apiezon N, Apiezon Products, M \& I Materials Ltd., Manchester, U.K.

${ }^{12}$ Stycast 1266, Emerson \& Cuming, Canton, MA.

${ }^{13}$ 83751B and 83556A, Agilent Technologies, Palo Alto, CA.

${ }^{14}$ 8757D, Agilent Technologies, Palo Alto, CA.

${ }^{15}$ Of course, pumping the whole He bath consumes a large amount of liquid $\mathrm{He}$. This problem can be solved by using an insert dewar or a $1 \mathrm{~K}$ pot. We omit these because of the simplicity of the design.

${ }^{16}$ CX-1030-SD, Lake Shore Cryotronics Inc., Westerville, OH.

${ }^{17}$ LTC-21, Neocera, Inc., Beltsville, MD.

${ }^{18}$ Run-to-run variation of the absolute value of $f_{B}^{0}$ is about $1 \mathrm{MHz}$, which corresponds to a change in the effective cavity height of $1 \mu \mathrm{m}$. In surface impedance measurements, this shift in $f_{B}^{0}$ can be included in the constant $C$ in Eq. (2) and is not a problem.

${ }^{19}$ R. I. Miller et al., Physica B 326, 296 (2003).

${ }^{20}$ J. Bardeen and M. J. Stephen, Phys. Rev. 140, A1197 (1965).

${ }^{21}$ M. Nohara, M. Isshiki, F. Sakai, and H. Takagi, J. Phys. Soc. Jpn. 68, 1078 (1999). 